\title{
Visible colour changes estimation in colorimetric determination of chromium (VI) using polymeric sensors
}

\author{
Nataliya A. Gavrilenko, Sergey V. Muravyov, Nadezhda V. Saranchina, Alexey V. Sukhanov \\ National Research Tomsk Polytechnic University, Pr. Lenina 30, 634050 Tomsk, Russia
}

\section{ABSTRACT}

The paper describes an application of a kind of optical analytical method, digital colour analysis (DCA), using colorimetric polymethacrylate sensors (optodes) in order to determine $\mathrm{Cr}(\mathrm{VI})$. The optodes are made of optically transparent polymethacrylate matrix (PMM) with 1.5-diphenylcarbazide immobilized. The developed optode can be used in determination of the analytes using solid phase spectrophotometry and calculating colour coordinates as functions of absorbance spectra. Also colour coordinates can be represented as basic colour (e.g. RGB) data after the optode image digitizing. Then one can determine the content of an analyte in a sample by an appropriate colour difference calculated for these coordinates. Experimental results of $\mathrm{Cr}(\mathrm{VI})$ determination in tap water show that the DCA relative standard deviation is $8-17 \%$ and recovery is $<12 \%$ at the range of determined concentrations $0.05-1.0$ $\mathrm{mg} \cdot \mathrm{L}^{-1}$. The characteristics are comparable with those of the solid phase spectrophotometry.

\section{Section: RESEARCH PAPER}

Keywords: colorimetric sensor; optode; chromium (VI); transparent polymeric matrix; immobilized reagents; digital colour analysis

Citation: Nataliya A. Gavrilenko, Sergey V. Muravyov, Nadezhda V. Saranchina, Alexey V. Sukhanov, Visible colour changes estimation in colorimetric determination of chromium (VI) using polymeric sensors, Acta IMEKO, vol. 5, no. 3, article 15, November 2016, identifier: IMEKO-ACTA-05 (2016)-03-15

Editor: Paolo Carbone, University of Perugia, Italy

Received July 4, 2016; In final form October 28, 2016; Published November 2016

Copyright: (C) 2016 IMEKO. This is an open-access article distributed under the terms of the Creative Commons Attribution 3.0 License, which permits unrestricted use, distribution, and reproduction in any medium, provided the original author and source are credited

Funding: This work was supported by Russian Science Foundation (project 14-19-00926)

Corresponding author: Sergey Muravyov, e-mail: muravyov@tpu.ru

\section{INTRODUCTION}

Chromium compounds are powerful oxidizing agents and tend to be irritating and corrosive. They appear to be well known toxic substances governing various health and ecological risks [1].

Trace levels of chromium can be determined by different analytical techniques most of which are based on atomic absorption spectrometry [2], inductively coupled plasmamass/atomic emission spectrometry [3], and high-performance liquid chromatography [4]. These methods, in spite of high sensitivity, need expensive instrumentation and skilled staff.

Optochemical sensors play an important part in industrial, environmental and clinical monitoring thanks to their low cost, possibility for miniaturization and great flexibility [5], [6]. Among different types of optochemical sensors, colorimetric sensors (optodes) are especially attractive because they recognize analytes through colour change that allows obtaining the visually observed and easily measurable analytical signal [7], [8]. The analytical signal measurement can be carried out using not only standard spectrophotometric equipment, but also the naked eye without the use of an expensive equipment. Naturally, the naked eye techniques cannot be as accurate as spectrophotometry. That is why the visible colour changes should be measured using different chromaticity coordinates (in RGB, XYZ, L*a*b*, and/or other systems) and parameters such as total colour difference (colour variation) $\Delta E$. Usually chromaticity parameters are calculated as functions of absorption or reflection spectra by means of computer programs intended for spectral data processing.

Nowadays, the measurement of colorimetric parameters has become easier due to a wide use of scanners, digital photo and video cameras, etc. [9]-[11]. An image of an optode is captured and transferred to a computer and its colour is interpreted using imaging software. This approach can be implemented also with transformation of an optode colour into an electric signal by means of a diode target array. It provides an opportunity for carrying out rapid chemical analysis in an industrial working environment and/or in the field. It also allows to design a 
reliable and easy-to-use measuring instrument that can be calibrated to be able to measure mass concentration of a whole series of substances. In the Tomsk Polytechnic University, such an analytical instrument has been created, which is called a digital colorimetric analyzer (DC analyzer) [12]. This portable instrument allows to measure colorimetric parameters of solid transparent optodes with high precision. The DC analyzer is controlled by a laptop or computer through a USB interface. Specially developed software enables the instrument calibration for different test systems. Due to compactness and ease of use, the DC analyzer can be used for rapid determinations in various practical domains.

In the present work, an application of the DC analyser, using colorimetric polymethacrylate sensors as optodes, is considered in order to determine $\mathrm{Cr}(\mathrm{VI})$. The optodes are made of optically transparent polymethacrylate matrix (PMM) with 1.5diphenylcarbazide immobilized. The developed optodes have been used in determining $\mathrm{Cr}$ (VI) using solid-phase spectrophotometry and calculating colour coordinates as functions of absorbance spectra. As an alternative approach, colour coordinates can be represented by means of RGB data after the optode image digitizing. Subsequent determination of the content of $\mathrm{Cr}(\mathrm{VI})$ in a sample has been carried out on the base of calculating an appropriate colour difference as a function of the RGB coordinates. Additionally, colorimetric parameters of the optode for Cr (VI) determination were measured by means of the DC analyser. Obtained outcomes are discussed in the present paper which is an extended version of the conference paper [13].

\section{METHODS}

\subsection{Materials}

The PMM is a specially created material containing functional groups which provide the ability to extract both the reagent and determined substance [14]. Transparent $10 \times 10 \mathrm{~cm}$ polymethacrylate plates, thickness $(0.60 \pm 0.04) \mathrm{mm}$, were prepared by the radical block polymerization of methacrylate and (alkyl)acrylates of alkaline (or alkaline earth) metals at the temperature $60-70{ }^{\circ} \mathrm{C}$ for 3-4 h. The plates were cut to platelets $6.0 \times 8.0 \mathrm{~mm}$ (weight ca. $0.05 \mathrm{~g}$ ) intended for analyses.

All reagents were of analytical grade and used as purchased without further purification. Deionized and distilled water was used in all experiments. The work solutions of $0.025-0.200 \%$ 1.5-diphenylcarbazide were prepared by dissolving precise loads in ethanol by heating in a water bath with subsequent diluting by distilled water. The stock solutions of metals $\left(1 \mathrm{mg} \cdot \mathrm{mL}^{-1}\right)$ were prepared by dissolving precise loads of their salts in 0.01 $\mathrm{M}$ acids. The work standard solutions of the required concentration were prepared by dilution of the stock solution in the day of the experiment. The required $\mathrm{pH}$ was adjusted using acid and alkali and controlled using an I-160 ionometer.

\subsection{Procedure}

The immobilization of 1.5-diphenylcarbazide (DPC) into the polymethacrylate matrix was performed by sorption from water-alcohol solution of DPC under bath conditions during 3$10 \mathrm{~min}$. The tailoring of the optimum conditions of the interaction of immobilized DPC with $\mathrm{Cr}(\mathrm{VI})$ are described in detail in [15]. Briefly, PMM with immobilized DPC was put into $50 \mathrm{ml}$ of $\mathrm{Cr}(\mathrm{VI})$ solution of different concentration and $\mathrm{pH}$ and stirred for 15-30 min. After that absorption spectra or absorbance of PMM was measured. Then the polymethacrylate matrix with the immobilized DPC was put into $50.0 \mathrm{~mL}$ of an analysed solution of definite concentration of $\mathrm{Cr}(\mathrm{VI})$ at a $\mathrm{pH} \approx$ 0 and stirred for $15 \mathrm{~min}$. After that absorption spectra of the PMM were measured. Then the colour coordinates $X, Y$ and $Z$ were calculated according to equations [16]:

$$
\begin{aligned}
& X=k \sum_{380}^{780} \tau(\lambda) S(\lambda) \bar{x}_{10}(\lambda) \Delta \lambda, \\
& Y=k \sum_{380}^{780} \tau(\lambda) S(\lambda) \bar{y}_{10}(\lambda) \Delta \lambda, \\
& Z=k \sum_{380}^{780} \tau(\lambda) S(\lambda) \bar{z}_{10}(\lambda) \Delta \lambda,
\end{aligned}
$$

where $k=\frac{100}{\sum_{380}^{780} S(\lambda) \bar{y}_{10}(\lambda) \Delta \lambda}, \quad \bar{x}_{10}(\lambda), \bar{y}_{10}(\lambda), \bar{z}_{10}(\lambda)$ are addition functions of CIE 1964 Supplementary Standard Observer, $\tau(\lambda)$ is transmittance of the PMM, $S(\lambda)$ is relative spectral power distribution of the CIE Standard Illuminant D65.

After that, chromaticity coordinates $x, y, z$ were found according to equations:

$$
\begin{aligned}
& x=X /(X+Y+Z), \\
& y=Y /(X+Y+Z), \\
& z=Z /(X+Y+Z),
\end{aligned}
$$

where the condition $x+y+z=1$ is valid.

Then the transition from the XYZ to the RGB system was made in accordance to the following equations:

$$
\begin{aligned}
& r=3.2405 x-1.5371 y-0.476 z, \\
& g=-0.9693 x+1.8760 y+0.041 z, \\
& b=0.0556 x-0.2040 y+1.0572 z .
\end{aligned}
$$

The conversion from the XYZ to the CIE $\mathrm{L}^{*} \mathrm{a}{ }^{*} \mathrm{~b}^{*}$ system was done as described by the following equations [17]:

$$
\begin{aligned}
& L^{*}=\left\{\begin{array}{ll}
116\left(Y / Y_{n}\right)^{\frac{1}{3}}-16 & \text { if } Y / Y_{n}>0.008856 \\
903.3\left(Y / Y_{n}\right) & \text { if } Y / Y_{n} \leq 0.008856
\end{array},\right. \\
& a^{*}=500\left[f\left(Y / Y_{n}\right)-f\left(Y / Y_{n}\right)\right], \\
& b^{*}=200\left[f\left(Y / Y_{n}\right)-f\left(Z / Z_{n}\right)\right],
\end{aligned}
$$

where $f(t)=\left\{\begin{array}{ll}t^{\frac{1}{3}} & \text { if } t>0.008856 \\ 7.787 t+16 / 116 & \text { if } t \leq 0.008856\end{array}\right.$,

$X_{n}, Y_{n}$ and $Z_{n}$ are values of coordinates of the white point of the system.

The total colour differences $\Delta E_{\mathrm{abs}}^{\mathrm{XYZ}}, \Delta E_{\mathrm{abs}}^{\mathrm{Lab}}$ and $\Delta E_{\mathrm{abs}}^{\mathrm{RGB}}$ for the chromaticity coordinates in XYZ, CIE L*a*b* and RGB colour systems calculated from absorption spectra were estimated according to the equations:

$$
\begin{aligned}
& \Delta E_{\mathrm{abs}}^{\mathrm{xYz}}=\left(\Delta x^{2}+\Delta y^{2}+\Delta z^{2}\right)^{1 / 2}, \\
& \Delta E_{\mathrm{abs}}^{\mathrm{Iab}}=\left(\Delta L^{2}+\Delta a^{2}+\Delta b^{2}\right)^{1 / 2}, \\
& \Delta E_{\mathrm{abs}}^{\mathrm{RGB}}=\left(\Delta r^{2}+\Delta g^{2}+\Delta b^{2}\right)^{1 / 2},
\end{aligned}
$$


where $\Delta x, \Delta y, \Delta q ; \Delta L, \Delta a, \Delta b$ and $\Delta r, \Delta g, \Delta b$ are the colour coordinate changes in the XYZ, CIE L*a*b* and RGB systems, respectively.

Visible colour changes of the PMM were estimated with digital imaging by means of a scanner and image processing software. The PMM image was captured and transferred to a computer and its colour was interpreted using imaging software where the colorimetric data in RGB format were related to the concentration of the analyte.

The total colour difference $\Delta E_{\text {scan }}$ for the scanned images of the PMM [18] evaluated by means of scanner and software Photoshop CS was estimated according to equation:

$$
\Delta E_{\text {scan }}=\left[\left(r_{0}-r\right)^{2}+\left(g_{0}-g\right)^{2}+\left(b_{0}-b\right)^{2}\right]^{1 / 2},
$$

where $r_{0}, g_{0}, b_{0}$ are the colour coordinates of samples after contact with a plain solution; $r, \mathrm{~g}, b$ are the colour coordinates of samples after contact with the solution containing the substance under determination.

Visible colour changes of the PMM were also estimated with the DC analyzer. For this aim, the PMM after contact with solution under determination was placed into the receiving bin of the analyzer and measurements were carried out. The measurement results were obtained as series of RGB coordinates and the corresponding colour difference $\Delta E_{\text {instr }}$ was calculated by formula similar to (16).

\subsection{Apparatus}

The absorption spectra and absorbencies of the PMM were recorded on the Schimadzu UV-mini-1240 (Schimadzu Corporation, Japan) and Spekol 21 (Carl Zeiss Jena, Germany) spectrophotometers. A non-modified polymethacrylate matrix was used as the reference sample.

As the desktop scanner the Hewlett Packard Scanjet 4400 C was used. The personal computer was Pentium II (333 MHz). The software packages were used as follows: Photoshop CS for image collection and taking colour intensities, Excel spreadsheet for general calculations and Origin 7.0 for plotting.

The colorimetric parameters were measured by means of the developed DC analyzer (instrumental error $<1 \%$, response time $<25 \mathrm{~ms}$ ).

The $\mathrm{pH}$ values were measured by the I-160 ionometer (NPO "Izmeritelnaya tekhnika", Russia) with a glass $\mathrm{pH}$-selective electrode. The ionometer had an absolute error $\pm 0.020 \mathrm{pH}$ and was calibrated at $25^{\circ} \mathrm{C}$ using buffer solutions with $\mathrm{pH} 1.00$ and 9.18 .

\section{RESULTS AND DISCUSSION}

Traditional colorimetric determination of chromium (VI) is based on the formation of the red-violet colored diphenylcarbazonate of chromium (III) complex as a result of interaction of DPC and $\mathrm{Cr}(\mathrm{VI})$ ions in acid media. The interaction includes the following two stages (Figure 1): redox reaction between chromium (VI) and 1.5-diphenyl-carbazide with formation of chromium (III) and diphenylcarbazonate (DPCO), and complex formation reaction between chromium (III) and DPCO.

The exact structure of this complex is not known but it appears to be a cationic complex [Cr(III)DPCO $]^{(3-\eta)+}$ in which an unknown number of protons $n$ are liberated [19].

Other colorimetric methods for chromium determination can be found in papers [20]-[23].

\section{1. $\mathrm{Cr}$ (VI) colorimetric determination using PMM}

The PMM with immobilized DPC after contact with Cr (VI) solution had red-violet colour due to the formation of the cationic complex. The absorption spectra of this complex in the PMM and scanned images of the PMM are presented in Figure 2. The absorption spectrum has a maximum at $545 \mathrm{~nm}$. The absorption at the wavelength $545 \mathrm{~nm}$ was taken as the analytical signal for solid phase spectrophotometric determination of $\mathrm{Cr}$ (VI) which was explained in more detail in [15].

\subsection{Acidity influence investigation}

Investigations of the influence of analyzed solution acidity on the analytical signal showed that maximum analytical signal
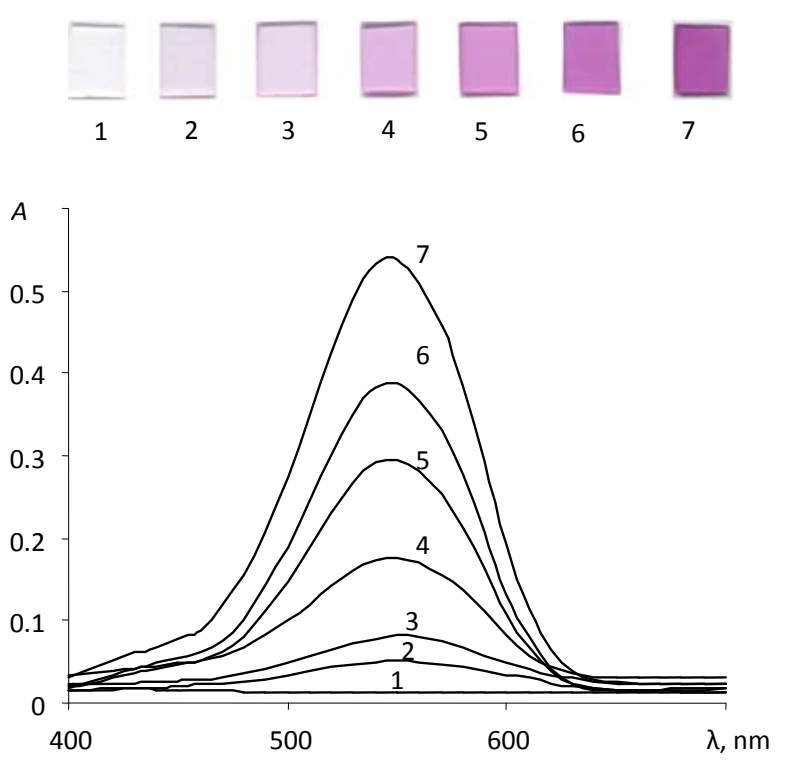

Figure 2. Absorption spectrum of PMM with immobilized DPC after contact with $\mathrm{Cr}(\mathrm{VI})$ solution with concentration, $\mathrm{mg} \cdot \mathrm{L}^{-1}$ : curve $1-0$; curve $2-0.05$; curve 3-0.10; curve 4-0.25; curve 5-0.50; curve 6-0.75; curve 7-1.00.

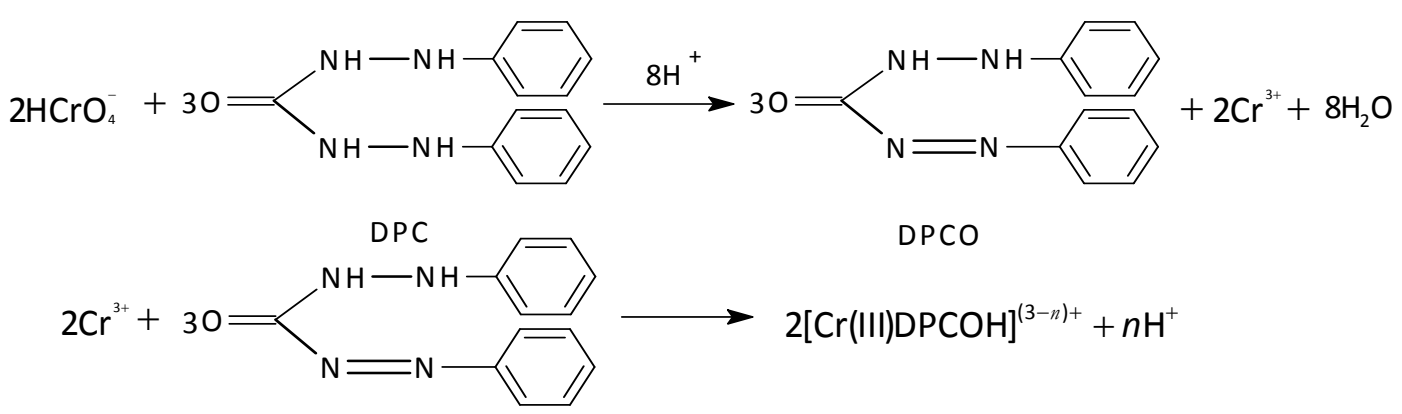

Figure 1. Scheme of $\mathrm{Cr}(\mathrm{VI})$ and DPC interaction. 
is reached at $\mathrm{pH} \approx 0$. Also, the nature of acid plays a significant role in shaping the analytical signal for solid phase spectrophotometric determination of $\mathrm{Cr}$ (VI). Therefore, sulphuric acid and orthophosphoric acid are not suitable for maintaining the medium acidity. Among hydrochloric acid and nitric acid the use of the former to maintain the medium acidity provided a maximum analytical signal which was stable during several hours, whereas the latter decreased the analytical signal and increased its stability up to several days. Thus, the hydrochloric acid was used in further work due to the maximum analytical signal.

\subsection{Interfering ions influence investigation}

Influence of interfering ions such as $\mathrm{Fe}$ (III), $\mathrm{Cu}$ (II), $\mathrm{Hg}$ (II), V (V), Co (III), Pb (II), Ni (II) and $\mathrm{Mn}$ (II) on the determination of $\mathrm{Cr}$ (VI) by using PMM with immobilized DPC was investigated. Ions of each of the interfering elements were added individually to a $50 \mathrm{mg} \cdot \mathrm{L}^{-1}$ chromium (VI) solution and relative error $\delta$ of $\mathrm{Cr}(\mathrm{VI})$ determination in presence of the interfering element ions was calculated according to equation

$\delta=\left[\left(A_{\Sigma}-A\right) / A\right] \cdot 100$,

where $A$ is an analytical signal from the PMM with immobilized DPC after contact with $\mathrm{Cr}(\mathrm{VI})$ solution without interfering ions; $A_{\Sigma}$ is the analytical signal after contact with the solution containing interfering ions. The interference investigation results are shown in Table 1.

\subsection{Calibration dependences}

The calibration dependences for Cr (VI) were obtained for different methods and analytical signals, namely: traditional solid phase spectrophotometry and absorbance maximum $A_{545}$; the solid phase spectrophotometry and total colour differences $\Delta E_{\mathrm{abs}}^{\mathrm{XYZ}}, \Delta E_{\mathrm{abs}}^{\mathrm{Iab}}$ and $\Delta E_{\mathrm{abs}}^{\mathrm{RGB}}$ computed by equations (14)-(16) on the base of the colour coordinates obtained from the absorption spectra; and the digital colour analysis (DCA) [10] with total colour differences $\Delta E_{\text {scan }}$ for scanned optode images and $\Delta E_{\text {instr }}$ measured by the DC analyzer. The concentration dependences for $\mathrm{Cr}$ (VI) are described by equations with high correlation coefficient values for all estimation methods of the visible colour changes. Analytical performance for the equations of the colour changes are presented in Table 2.

Graphical dependencies of the analytical signal versus concentration of $\mathrm{Cr}(\mathrm{VI})$ in the analyzed solution are shown in Figure 3. One can see in Figure 3 that the slope of the calibration line $\Delta E_{\mathrm{abs}}^{\mathrm{RGB}}$ is greater than that of the calibration line

Table 1. Influence of interfering ions.

\begin{tabular}{lcc}
\hline Ions & Concentration ratios & $\boldsymbol{\delta}, \%$ \\
\hline \multirow{2}{*}{$\mathrm{Fe}$ (II) } & 10 & 5 \\
& 50 & 7 \\
\hline \multirow{2}{*}{$\mathrm{Cu}(\mathrm{II})$} & 10 & 5 \\
& 50 & 10 \\
\hline $\mathrm{V}(\mathrm{V})$ & 10 & 5 \\
& 50 & 13 \\
$\mathrm{Hg}$ (II) & 10 & 5 \\
\hline \multirow{2}{*}{$\mathrm{Mn}(\mathrm{II})$} & 50 & 13 \\
\hline $\mathrm{Co}$ (III) & 10 & 5 \\
\hline $\mathrm{Pb}$ (II) & 50 & 13 \\
\hline $\mathrm{Ni}$ (II) & 50 & 5 \\
\hline
\end{tabular}

Table 2. Analytical performance of the $\mathrm{Cr}(\mathrm{VI})$ colorimetric sensor.

\begin{tabular}{llll}
\hline $\begin{array}{c}\text { Analytical } \\
\text { signal }\end{array}$ & \multicolumn{1}{c}{ Calibration equation } & $\boldsymbol{R}^{1}$ & $\mathbf{R D C}, \mathbf{m g} \cdot \mathbf{L}^{-1}$ \\
\hline$A_{545}$ & $0.003+0.525 \cdot C_{\mathrm{Cr}(\mathrm{VI})}$ & 0.998 & $0.05-1.00$ \\
$\Delta E_{\mathrm{abs}}^{\mathrm{RGB}}$ & $0.002+0.167 \cdot C_{\mathrm{Cr}(\mathrm{VI})}$ & 0.999 & $0.05-1.00$ \\
$\Delta E_{\mathrm{abs}}^{\mathrm{Lab}}$ & $0.4+47.8 \cdot C_{\mathrm{Cr}(\mathrm{VI})}$ & 0.999 & $0.05-1.00$ \\
$\Delta E_{\mathrm{abs}}^{\mathrm{xr}}$ & $0.003+0.103 \cdot C_{\mathrm{Cr}(\mathrm{VI})}$ & 0.998 & $0.05-1.00$ \\
$\Delta E_{\mathrm{scan}}$ & $5.63+208.29 \cdot C_{\mathrm{Cr}(\mathrm{VI})}$ & 0.997 & $0.05-1.00$ \\
$\Delta E_{\text {instr }}$ & $77.24 \cdot C_{\mathrm{Cr}(\mathrm{VI})}$ & 0.992 & $0.05-1.00$ \\
\hline${ }^{1} R$ is a correlation coefficient & & \\
${ }^{2} \mathrm{RDC}$ is a range of determined concentrations & &
\end{tabular}

$\Delta E_{\mathrm{abs}}^{X Y Z}$. It means that the use of RGB colour coordinates when computing the total colour difference for absorbance spectrum data provides a better sensitivity compared to XYZ coordinates.

It should be noticed that, in the case of $\mathrm{Cr}$ (VI), the calibration dependence for $\Delta E_{\text {scan }}$ appears to have quadratic character. The calibration dependence for $\Delta E_{\text {instr }}$ could be described by both a second degree polynomial and a linear equation. As soon as the correlation coefficients for the two options were equal to each other, for the sake of simplicity, the linear equation was selected.

Precision of the procedure was expressed as the relative standard deviation (RSD) for the determination of $0.1 \mathrm{mg} \cdot \mathrm{L}^{-1}$ of $\mathrm{Cr}(\mathrm{VI})$. Values of range of determined concentrations (RDC), as well as of RSD, were obtained under conditions of reproducibility for a number of determinations $n=5$ according to ISO 5725-1994.

Expressed in percents $R S D$ was estimated by the formula

$R S D=\frac{1}{\bar{C}} \sqrt{\frac{1}{n-1} \sum_{i=1}^{n}\left(C_{i}-\bar{C}\right)^{2}}$,

where $n$ is the number of determinations (optodes); $C_{i}$ is the result of $i$-th concentration determination; and $\bar{C}$ is the arithmetic mean of the $n$ determination results.

Expressed in percents recovery $\mathcal{Q}$ was estimated by the formula

$Q=\frac{Q_{\text {added }}-Q_{\text {found }}}{Q_{\text {added }}}$,

where $Q_{\text {added }}$ is introduced addition content and $Q_{\text {found }}$ is found addition content as an averaged value of $n$ determinations.

The accuracy and precision of $\mathrm{Cr}(\mathrm{VI})$ determination results were verified by the standard addition method using drinking water (Table 3). One can see from Table 3 that the characteristics of accuracy and precision for analytical signals and $\Delta E_{\text {scan }}$ of the digital colour analysis are comparable with those of the solid phase spectrophotometry ( $A_{545}$ and $\left.\Delta E_{\text {abs }}\right)$.

\section{CONCLUSION}

The proposed colorimetric sensor on the base of the PMM with immobilized DPC can be successfully used for the determination of $\mathrm{Cr}(\mathrm{VI})$ by both solid phase spectrophotometry and digital colour analysis (DCA). Different analytical signals based on estimation of visible colour changes were studied. Metrological performance of the DCA method was shown to be comparable with those of the solid phase spectrophotometry. 

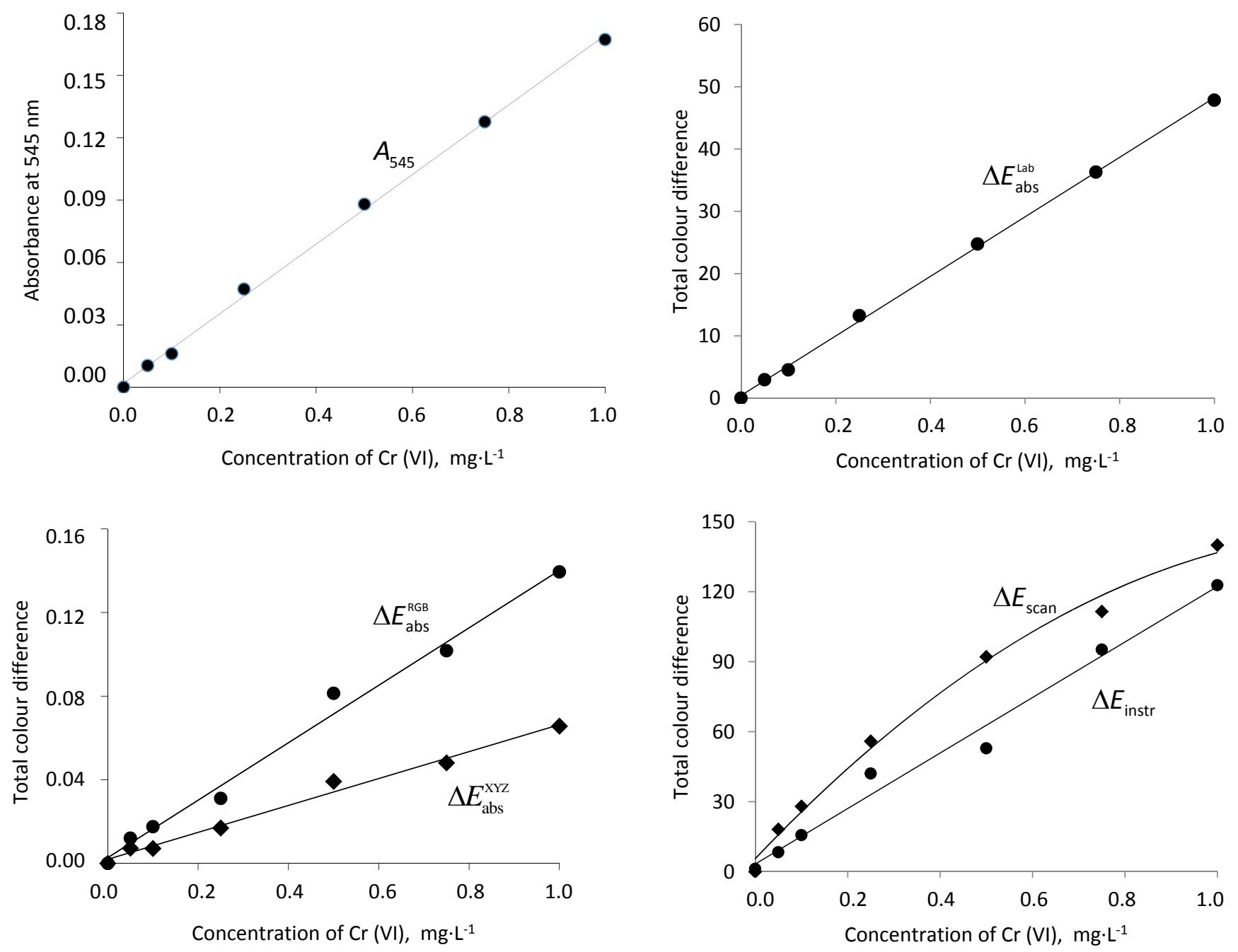

Figure 3. Calibration dependences for determination of $\mathrm{Cr}(\mathrm{VI})$ obtained for estimation of visible colour changes using the analytical signals: $A_{545}, \Delta \mathbb{E}_{\mathrm{abs}}^{\mathrm{rg}}$, $\Delta E_{\mathrm{abs}}^{\mathrm{ab}}, \Delta E_{\mathrm{abs}}^{\mathrm{RoB}}, \Delta E_{\text {scan }}$ and $\Delta E_{\text {instr. }}$

Table 3. Determination of $\mathrm{Cr}(\mathrm{VI})$ in tap water (sample size $n=5$, confidence level $P=0.95$ ).

\begin{tabular}{|c|c|c|c|c|c|c|}
\hline Technique & Analytical s & & Added, $\mathrm{mg} \cdot \mathrm{L}^{-1}$ & Found, $\mathrm{mg} \cdot \mathrm{L}^{-1}$ & $\mathrm{RSD}^{1}, \%$ & Recovery, \% \\
\hline \multirow{12}{*}{$\begin{array}{l}\text { Solid-phase } \\
\text { spectrophotometry }\end{array}$} & \multirow{3}{*}{\multicolumn{2}{|c|}{$A_{545}$}} & 0.070 & $0.065 \pm 0.016$ & 15 & -7 \\
\hline & & & 0.300 & $0.29 \pm 0.03$ & 7 & -3 \\
\hline & & & 0.600 & $0.601 \pm 0.023$ & 2 & 0.2 \\
\hline & \multirow{9}{*}{ Colour coordinates } & \multirow{3}{*}{$\Delta E_{\mathrm{abs}}^{\mathrm{RGB}}$} & 0.070 & $0.066 \pm 0.008$ & 14 & -6 \\
\hline & & & 0.300 & $0.29 \pm 0.02$ & 8 & -3 \\
\hline & & & 0.600 & $0.63 \pm 0.05$ & 8 & 5 \\
\hline & & \multirow{3}{*}{$\Delta E_{\mathrm{abs}}^{\mathrm{Lab}}$} & 0.070 & $0.068 \pm 0.008$ & 14 & -3 \\
\hline & & & 0.300 & $0.280 \pm 0.023$ & 8 & -5 \\
\hline & & & 0.600 & $0.62 \pm 0.05$ & 8 & 3 \\
\hline & & \multirow{3}{*}{$\Delta E_{\mathrm{abs}}^{\mathrm{xrz}}$} & 0.070 & $0.070 \pm 0.013$ & 21 & 0.2 \\
\hline & & & 0.300 & $0.29 \pm 0.03$ & 12 & -3 \\
\hline & & & 0.600 & $0.63 \pm 0.04$ & 7 & 4 \\
\hline \multirow{6}{*}{ Digital colour analysis } & \multirow{3}{*}{\multicolumn{2}{|c|}{$\Delta E_{\text {scan }}$}} & 0.070 & $0.069 \pm 0.012$ & 20 & -2 \\
\hline & & & 0.300 & $0.31 \pm 0.03$ & 11 & 2 \\
\hline & & & 0.600 & $0.59 \pm 0.06$ & 11 & -2 \\
\hline & \multirow{3}{*}{\multicolumn{2}{|c|}{$\Delta E_{\text {instr }}$}} & 0.070 & $0.066 \pm 0.005$ & 8 & -6 \\
\hline & & & 0.300 & $0.34 \pm 0.05$ & 17 & 12 \\
\hline & & & 0.600 & $0.65 \pm 0.08$ & 14 & 9 \\
\hline
\end{tabular}

\footnotetext{
${ }^{1} \mathrm{RSD}$ is a relative standard deviation
} 
The DCA for determination of not only Cr (VI) but also a wide spectrum of other elements and/or substances can be implemented using an office scanner with graphic software or the DC analyzer. Regardless of the implementation mode, it can be applied as a highly sensitive method of chemical analysis in both laboratory and field conditions. Use of the DC analyzer facilitates field measurements by means of the DCA due to its compactness.

\section{ACKNOWLEDGEMENT}

This work was supported by Russian Science Foundation, project 14-19-00926.

\section{REFERENCES}

[1] EPA/630/P-02/004F, Generic Ecological Assessment Endpoints (GEAE) for Ecological Risk Assessment, U.S. Environmental Protection Agency, Washington, DC, October 2003.

[2] Z. Tahmasebi, S.S.H. Davarani, Selective and sensitive speciation analysis of $\mathrm{Cr}$ (VI) and $\mathrm{Cr}$ (III), at sub- $\mu \mathrm{g} \mathrm{L}^{-1}$ levels in water samples by electrothermal atomic absorption spectrometry after electromembrane extraction, Talanta 161 (2016) pp. 640-646.

[3] R. Nageswara Rao, M.V.N. Kumar Talluri, An overview of recent applications of inductively coupled plasma-mass spectrometry (ICP-MS) in determination of inorganic impurities in drugs and pharmaceuticals, J. Pharm. Biomed. Anal. 43(1) (2007) pp. 1-13.

[4] P. Jin, X. Liang, L. Xia, F. Jahouh, R. Wang, Y. Kuang, X. Hu, Determination of 20 trace elements and arsenic species for a realgar-containing traditional Chinese medicine Niuhuang Jiedu tablets by direct inductively coupled plasma-mass spectrometry and high performance liquid chromatography-inductively coupled plasma-mass spectrometry, J. Trace Elem. Med. Biol. 33 (2016) pp. 73-80.

[5] C. McDonagh, C.S. Burke, B.D. McCraith, Optical chemical sensors, Chem. Rev. 108(2) (2008) pp. 400-422.

[6] R. Narayanaswamy, O.S. Wolfbeis (Eds.), Optical sensors, Industrial, environmental and diagnostic applications, Springer, 2004, ISBN 978-3662-09111-1

[7] N. Kaur, S. Kumar, Colorimetric metal ion sensor, Tetrahedron 67 (2011) pp. 9233-9264.

[8] N. Sato, M. Mori, H. Itabashi, Cloud point extraction of Cu (II) using a mixture of triton X-100 and dithizone with a salting-out effect and its application to visual determination, Talanta 117 (2013) pp. 376-381.

[9] S.V. Khimchenko, L.P. Eksperiandova, Comparison of analytical potentials of detection versions in chromaticity rapid analysis using portable instruments, J. Anal. Chem. 67(8) (2012) pp. 701705.

[10] N.A. Gavrilenko, S.V. Muravyov, S.V. Silushkin, A.S. Spiridonova, Polymethacrylate optodes: A potential for chemical digital colour analysis, Measurement 51 (2014) pp. 464-469.

[11] J.R. Askim, M. Mahmoudi, K.S. Suslick, Optical sensor arrays for chemical sensing: the optoelectronic nose, Chem. Soc. Rev. 42(22) (2013) pp. 8649-8682.

[12] S.V. Muravyov, A.S. Spiridonova, Gavrilenko N.A., P.F. Baranov, L.I. Khudonogova, A digital colorimetric analyzer for chemical measurements on the basis of polymeric optodes, Instrum. Exp. Tech. 59(4) (2016) pp. 592-600.

[13] N. Saranchina, N. Gavrilenko, A. Sukhanov, S. Muravyov "Colorimetric polymer sensor for determination of chromium (VI): comparison of estimation methods of the visible colour changes", Proc. of $21^{\text {st }}$ IMEKO World Congress, Aug. 30-Sept. 4, 2015, Prague, Czech Republic.

[14] N.A. Gavrilenko, G.M. Mokrousov, "The indicator sensitive material for determination of microquantities of substances", Patent 2272284 (RU), 2004.

[15] N.V. Saranchina, I.V. Mikheev, N.A. Gavrilenko, M.A. Proskurnin, M.A. Gavrilenko, Determination of chromium (VI) using 1.5-diphenylcarbazide immobilized in a polymethacrylate matrix, Analitika i kontrol 18(1) (2014) pp. 105-111 (in Russian).

[16] D. Jadd, G. Wyszecki, Colour in Business, Science and Industry, New York, Wiley, 1975, ISBN: 0471452122.

[17] A. Ford, A. Roberts. Colour space conversions. Westminster University, London, 1998, http://www.poynton.com/PDFs/ coloureq.pdf

[18] S.V. Murav'ev, N.A. Gavrilenko, A.S. Spiridonova, S.V. Silushkin, "Method of determining amount of analysed substance from colour scale", Patent 2428663 (RU), 2011.

[19] Y.M. Scindia, A.K. Pandey, A.V.R. Reddy, S.B. Manohar, Chemically selective membrane optode for $\mathrm{Cr}$ (VI) determination in aqueous samples, Anal. Chim. Acta 515 (2004) pp. 311-321.

[20] Xiaoyan Wu, Yunbo Xu, Yangjun Dong, Xue Jiang, Ningning Zhu, Colorimetric determination of hexavalent chromium with ascorbic acid capped silver nanoparticles, Anal. Methods 5 (2013) pp. 560-565.

[21] Jing Li, Hui Wei, Shaojun Guo, Erkang Wang, Selective, peroxidase substrate based "signal-on" colorimetric assay for the detection of chromium (VI), Anal. Chim. Acta 630 (2008) 181185.

[22] D. Kim, J. Om, Direct spectroscopic determination of aqueous phase hexavalent chromium, UJES 1(1) (2013) pp. 1-4.

[23] P. Parmar, A.K. Pillai, V.K. Gupta, An improved colorimetric determination of micro amounts of chromium (VI) and chromium (III) using p-aminoacetophenone and phloroglucinol in different samples, J. Anal. Chem. 65(6) (2010) pp. 582-587. 